\title{
Spatially Confined Defects Induce Chemical Functionality of Anisotropic $\mathrm{TiO}_{2}$ Rods
}

\author{
B.M. Rabatic, ${ }^{*}$ N.M. Dimitrijevic, ${ }^{* *}$ R.E. Cook, *** Z.V. Saponjic, ${ }^{* *}$ and T. Rajh** \\ * Center for Nanoscale Materials, Argonne National Laboratory, 9700 South Cass Avenue, Argonne, \\ IL 60439 \\ ** Chemistry Division, Argonne National Laboratory, 9700 South Cass Avenue, Argonne, IL 60439 \\ *** Materials Science Division, Argonne National Laboratory, 9700 South Cass Avenue, Argonne, \\ IL 60439
}

High-resolution transmission electron microscopy techniques were used to characterize the fine structure of anisotropic titanium dioxide $\left(\mathrm{TiO}_{2}\right)$ nanoparticles. These particles were synthesized by a surfactant-free hydrothermal procedure to form anatase $\mathrm{TiO}_{2}$ particles. The particles are single crystals and have a rod-like appearance with lengths up to $300 \mathrm{~nm}$ and widths up to 50nm. From our investigation, we identified site-specific defects located at the tips of the nanorods. These defects, referred to as "corner defects," are related to the size and shape of features on the nanoparticle.

In the present example, the small dimension and high radius of curvature found at the nanorod tips creates the defect by constraining the atomic arrangement of titanium (Ti) atoms. This confinement, occurring within about five atomic layers from the tip, imparts an under-coordinated atomic character to the Ti metal sites and results in lengthening of the Ti-Ti atom distances along the [100] direction of the nanocrystal. Utilizing fast Fourier transformation (FFT) analysis techniques to quantify the lattice spacing, we find an increase of approximately $0.3 \AA$ to the Ti-Ti bond length of the defect area when compared to the bulk material. At the defect, the Ti-Ti spacing is $3.96 \pm 0.20 \AA$ whereas the non-defective spacing measures $3.70 \pm 0.19 \AA$.

We found that the undercoordinated defect sites are prone to direct chemical functionalization and preferentially bind the bidentate molecule dopamine (3,4-dihydroxyphenylethamine). Upon exposure to dopamine, relaxation of the extended corner defect spacing, to values associated with bulk material, occurs. We hypothesize that binding of dopamine is the result of optimal matching of the enediol groups of dopamine to the undercoordinated titanium atoms at the defect site and causes the atomic reorganization. A similar defect healing effect is also known to occur in spherical nanoparticles of $\mathrm{TiO}_{2}$ upon complexing with dopamine [1]. By selectively modifying the tip surfaces of these nanoparticles with biotin, we are able to induce the tip-to-tip serial assembly of these biohybrid nanocomposites by conjugation with avidin [2]. This reactivity observed at the nanorod ends is reminiscent of the selectivity found with organic and biological self-organizing systems.

\section{References}

[1] T. Rajh, L.X. Chen, K. Lukas, T. Liu, M.C. Thurnauer, and D.M. Tiede, J. Phys. Chem. 106 (2002) 10543.

[2] N.M. Dimitrijevic, Z.V. Saponjic, B.M. Rabatic, and T. Rajh, J. Am. Chem. Soc. 127 (2005) 1344.

[3] Supported by the U.S. Department of Energy, Office of Basic Energy Sciences, Division of Materials Science, under contract W-31-109-Eng-38. 


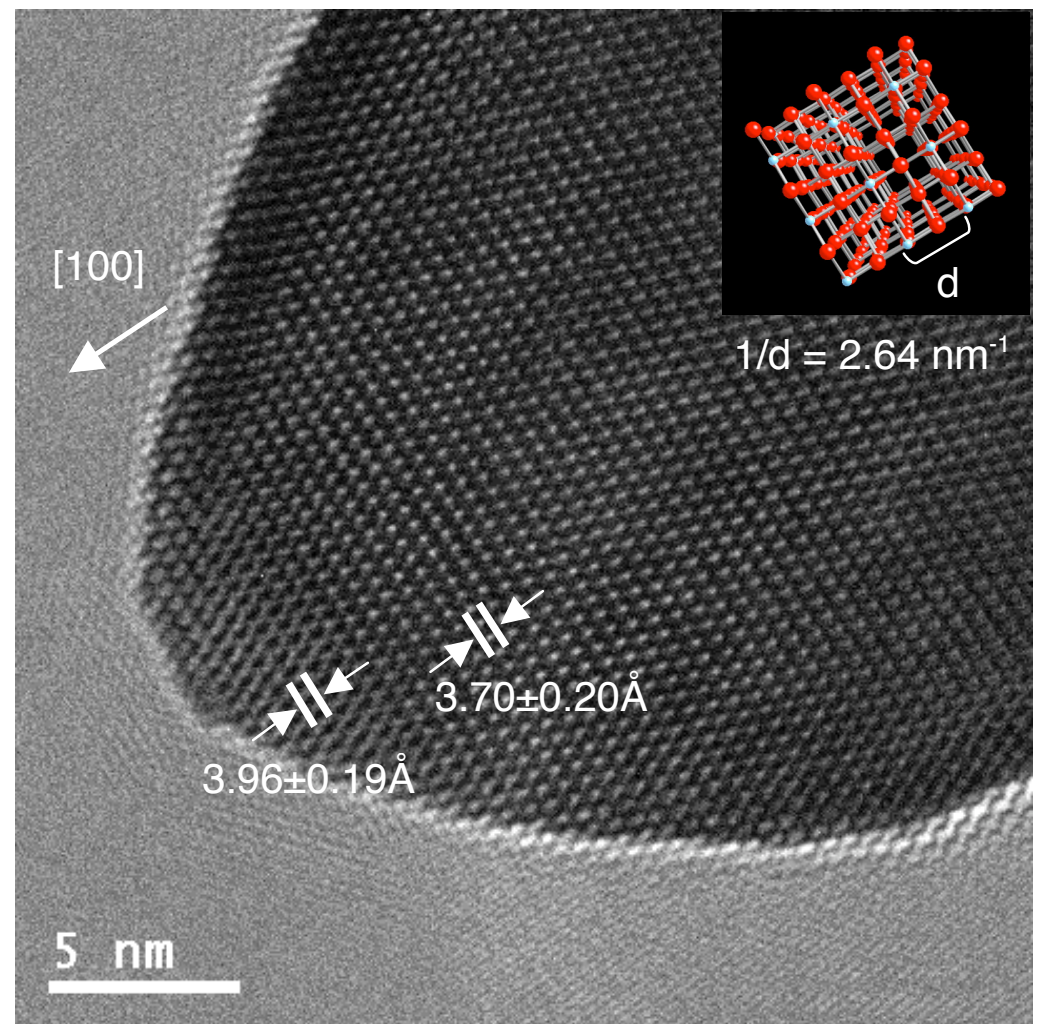

Fig. 1. HRTEM image of a $\mathrm{TiO}_{2}$ nanorod showing the extended [001] spacing at the defect site.



Fig. 2. Tip-to-tip self-assembly of $\mathrm{TiO}_{2}$ nanorod bio-hybrid nanocomposites conjugated through the glycoprotein avidin. 